\title{
A NOTE ON THE ASYMPTOTIC BEHAVIOR OF AN INTEGRAL EQUATION
}

\author{
CAROL SHILEPSKY ${ }^{1}$
}

ABstract. Assume the existence and boundedness of a solution to an integral equation. Conditions are found which ensure the solution has a limit at infinity.

1. Introduction. Consider the integral equation

$$
x^{\prime}(t)+\int_{-\infty}^{\infty} g(x(t-\xi)) d A(\xi)=f(t)
$$

where $g, A$ and $f$ are prescribed real functions, and $x$ is a bounded solution of $(1.1)$ on $(-\infty, \infty)$. We use NBV to denote normalized bounded variation, LAC for absolutely continuous on any compact interval of $(-\infty, \infty), V\left(A,\left[t_{1}, t_{2}\right]\right)$, the total variation of $A$ on $\left[t_{1}, t_{2}\right]$, and $V(A)=$ $V(A,(-\infty, \infty))$. Consider the following hypotheses.

$H(f): f \in L^{\infty}(-\infty, \infty), \lim _{t \rightarrow \infty} f(t)=f(\infty)$ exists.

$H(g): g \in C(-\infty, \infty), S=\{c \mid g(c) A(\infty)=f(\infty)\}$ is nonempty and contains no interval.

$$
\begin{aligned}
& H^{\prime}(g): g \in C(-\infty, \infty), S \text { contains exactly one point. } \\
& H(A): A(t)=A_{1}(t)+A_{2}(t), A_{1}(t)=0(-\infty<t \leqq 0), \\
& \quad A_{1}(t)=\rho_{1}>0(0<t<\infty), \quad A_{2}(t) \in \mathrm{NBV}(-\infty, \infty),
\end{aligned}
$$

and $V\left(A_{2}\right)=\rho_{2}<\rho_{1}$.

THEOREM 1. Let $H(f), H(g)$ and $H(A)$ hold. Let $x(t) \in \mathrm{LAC}(-\infty, \infty) \cap$ $L^{\infty}(-\infty, \infty)$, let $x^{\prime}(t)$ exist for each $t$ and let $x(t)$ satisfy $(1.1)$ on $(-\infty, \infty)$. Then

for some $c \in S$.

$$
\lim _{t \rightarrow \infty} x(t)=c, \quad \lim _{t \rightarrow \infty} x^{\prime}(t)=0
$$

Received by the editors June 11, 1971 and, in revised form, July 23, 1971. AMS 1970 subject classifications. Primary 45M05, 45G99; Secondary 45D05.

Key words and phrases. Integral equation, stability theory, Volterra equation, asymptotic behavior, bounded solution.

1 This research was supported by the U.S. Army Research Office, Durham.

(c) American Mathematical Society 1972 
THEOREM 2. Let $H(f), H^{\prime}(g)$ and $H(A)$ hold. Let $x(t) \in \mathrm{LAC}(-\infty, \infty) \cap$ $L^{\infty}(-\infty, \infty)$ satisfy $(1.1)$ a.e. on $(-\infty, \infty)$. Then

for $c \in S$.

$$
\lim _{t \rightarrow \infty} x(t)=c, \quad \lim _{t \rightarrow \infty}\left[\underset{t \leqq r<\infty}{\operatorname{ess} \sup }\left|x^{\prime}(\tau)\right|\right]=0
$$

Theorem 1 was obtained by Levin and Shea [2] under the additional hypothesis that $g$ is either strictly increasing or strictly decreasing. Theorem 2 assumes that $x$ is a solution of (1.1) only a.e. on $(-\infty, \infty)$ and was obtained independently by Levin and Shea and by the author. A proof appears in [1].

It is shown in [1] that the requirement $\rho_{2}<\rho_{1}$ is necessary in $H(A)$. It is obvious that the requirement, $x$ a bounded solution, is necessary in Theorems 1 and 2. For if $g(x)=-x, A(t)=0(-\infty<t \leqq 0), A(t)=1$ $(0<t<\infty), f(t) \equiv 0$, then $c=0$ and (1.1) reduces to $x^{\prime}(t)-x(t)=0$ which has $x(t)=e^{t}$ as a solution.

For further discussion of (1.1) and references to applications in ordinary differential equations and Volterra equations see [1] and [2].

2. Proof of Theorem 1. We first note that $g(c)$ is the same for all $c$ in $S$. Let $x(t) \in \operatorname{LAC}(-\infty, \infty) \cap L^{\infty}(-\infty, \infty)$ satisfy (1.1). Let

$$
\alpha=\sup _{-\infty<t<\infty}|g(x(t))-g(c)|
$$

where $c \in S, \bar{\alpha}=\lim \sup _{t \rightarrow \infty}|g(x(t))-g(c)|$. Suppose $\bar{\alpha}>0$. Let $\varepsilon>0$, and, using $\rho_{2}<\rho_{1}$, let $T_{\varepsilon}$ be chosen such that

$$
\begin{gathered}
\left(\rho_{2}-\rho_{1}\right) \bar{\alpha}+\left(\rho_{2}+\rho_{1}+\alpha+1\right) \varepsilon<-d \\
\quad \text { for some positive constant } d, \\
\sup _{t>T_{\varepsilon}}|g(x(t))-g(c)|<\bar{\alpha}+\varepsilon, \\
V\left(A_{2 .},\left[T_{\varepsilon}, \infty\right)\right)<\varepsilon, \text { and } r \\
\sup _{t>T_{\varepsilon}}|f(t)-f(\infty)|<\varepsilon .
\end{gathered}
$$

By definition of $\bar{x}$, there exist $\left\{t_{n}\right\}_{n=1}^{\infty}$ and $\left\{\varepsilon_{n}\right\}_{n=1}^{\infty}$ with $t_{1}>2 T_{\varepsilon}, \varepsilon_{1}<\varepsilon$ such that $t_{n} \rightarrow \infty(n \rightarrow \infty), \varepsilon_{n} \downarrow 0(n \rightarrow \infty)$ and either

$$
\bar{\alpha}-\varepsilon_{n}<g\left(x\left(t_{n}\right)\right)-g(c)<\bar{\alpha}+\varepsilon_{n}, \quad \text { or }
$$

(2.3) $-\bar{\alpha}-\varepsilon_{n}<g\left(x\left(t_{n}\right)\right)-g(c)<-\bar{x}+\varepsilon_{n} \quad(n=1,2, \cdots)$.

Suppose (2.2) holds. From (1.1) and $H(A)$ we have

$$
x^{\prime}(t)+\rho_{1} g(x(t))=-\int_{-\infty}^{\infty} g(x(t-\xi)) d A_{2}(\xi)+f(t)
$$


and using $f(\infty)=g(c) A(\infty)=g(c)\left(\rho_{1}+A_{2}(\infty)\right)$,

$$
\begin{aligned}
x^{\prime}(t)+\rho_{1}(g(x(t))-g(c)) & \\
& =-\int_{-\infty}^{\infty}(g(x(t-\xi))-g(c)) d A_{2}(\xi)+f(t)-f(\infty) .
\end{aligned}
$$

Let $t=t_{n}$. By (2.1) and (2.2) we have

$$
\begin{aligned}
x^{\prime}\left(t_{n}\right)+\rho_{1}\left(\bar{\alpha}-\varepsilon_{n}\right) & \\
& \leqq \\
& \left.\leqq-\int_{-\infty}^{T_{\varepsilon}}-\int_{T_{\varepsilon}}^{\infty}\right\}\left(g\left(x\left(t_{n}-\xi\right)\right)-g(c)\right) d A_{2}(\xi)+f\left(t_{n}\right)-f(\infty) \\
& \leqq(\bar{\alpha}+\varepsilon) \rho_{2}+\alpha \varepsilon+\varepsilon,
\end{aligned}
$$

and hence by $(2.1)$,

$$
x^{\prime}\left(t_{n}\right)<-d .
$$

By a similar argument, $t>t_{n}$ and $x(t)=x\left(t_{n}\right)$ impiy $x^{\prime}(t)<-d$. Hence $t>t_{n}$ and $x$ continuous imply $x(t)<x\left(t_{n}\right)$. In particular,

$$
-\|x\|_{\infty} \leqq x\left(t_{n+1}\right)<x\left(t_{n}\right) \text {. }
$$

Thus there exists $x^{*}$ such that $x\left(t_{n}\right) \downarrow x^{*}$. By $(2.2), g\left(x^{*}\right)-g(c)=\bar{\alpha}$. Since $g \in C(-\infty, \infty)$, there exists $x^{* *}>x^{*}$ such that, for $x^{*}<x(t)<x^{* *}$, $\bar{\alpha}-\varepsilon<g(x(t))-g(c)<\bar{\alpha}+\varepsilon$. Choose $N$ such that $x^{*}<x\left(t_{N}\right)<x^{* *}$ and hence for $t>t_{N}, x^{*}<x(t)<x^{* *}$. Then as in the proof of (2.4), we have $t>t_{N}$ implies $x^{\prime}(t)<-d$. This contradicts $x \in L^{\infty}$, hence (2.2) is impossible.

Similarly (2.3) is impossible, $\bar{\alpha}=0$ and $\lim _{t \rightarrow \infty} g(x(t))=g(c)$, where $g(c)=g^{*}$ is independent of $c \in S$. Then $\lim _{t \rightarrow \infty} g(x(t))=g^{*}$ implies $x(t)$ approaches a value of $x$ such that $g(x)=g^{*}$. That is, $\lim _{t \rightarrow \infty} x(t)=c$ for some $c \in S$. From $(1.1)$ we then have $\lim _{t \rightarrow \infty} x^{\prime}(t)=0$.

\section{BIBLIOGRAPHY}

1. J. J. Levin and D. F. Shea, Asymptotic behavior of the bounded solutions of some functional equations, Contributions to Nonlinear Functional Analysis, U.S. Army Math. Res. Center, Madison, Wis., 197 !.

2. - On the asymptotic behavior of the bounded solutions of some integral equations, J. Math. Anal. Appl. (to appear).

Department of Mathematics, University of Wisconsin, Madison, Wisconsin 53706

Current address: Box 2102, State University, Arkansas 72467 